Discussion Points

1 How would you begin to develop the necessary networks to deliver an effective multidisciplinary sexual health service to women with IBD?

2 Many areas do not currently have access to DEXA scanning. Discuss how you might risk manage this situation in patients with IBD who would ideally have a DEXA scan for assessment of osteoporosis.

3 What issues would you cover in a preconceptual counselling session in a woman with CD?

4 The issue of VTE is a contentious one in IBD. It appears unclear whether this is an inherent difficulty or acquired through inactivity or disease. Outline the form your discussion with a patient with well-controlled UC who wished to use COC might take.

\title{
Questions for the Inflammatory Bowel Disease and Contraception Guidance
}

The following questions and answers have been developed by the FFPRHC Education Committee.

Indicate your answer by ticking the appropriate box for each question

True False

1 In general, women with IBD requiring EC should be advised to take an additional $750 \mu \mathrm{g}$ (microgrammes) levonorgestrel tablet over and above the usual dose.

2 There is no evidence that the combined pill causes any deterioration in IBD activity.

3 The POP should be discontinued 4-6 weeks prior to elective surgery in women with IBD.

4 It would be usual to recommend a method more effective than barrier contraception for a woman using methotrexate therapy.

5 At an early stage, women with IBD should be made aware that abdominal surgery for their disease may lead to subsequent subfertility.

6 Women should be advised that the efficacy of oral contraception is unlikely to be reduced by large bowel disease.

7 Women with CD may be at increased risk of preterm labour.

8 Women with IBD are at an increased risk of osteopenia or osteoporosis, therefore if use of DMPA is being considered measurement of BMD is recommended.

9 There is no known additional increased risk of pelvic infection in women with IBD who choose to use an IUD.

10 Laparoscopic sterilisation would be a suitable method of contraception for a woman with IBD who had had previous pelvic or abdominal surgery.

\begin{tabular}{|c|c|c|c|c|}
\hline Answers & & & & \\
\hline 1 False & 5 & True & 8 & True \\
\hline 2 True & 6 & True & 9 & True \\
\hline 3 False & 7 & True & 10 & False \\
\hline
\end{tabular}

\section{Visit the Faculty Website at: www.ffprhc.org.uk}

